\title{
Modelling and an SS Approach for the Parallel Production Lines Scheduling Problem
}

\author{
Hui-Zhi Ren, Han Xu \\ School of Mechanical Engineering, \\ Shenyang University of Technolgy \\ Shenyang, 110870, P.R.China \\ E-mail: renhuizhi@126.com, 11592424136@qq.com
}

\begin{abstract}
A special parallel machine scheduling problem is studied and formulated into a mixed integer programming model, considering setup time and time window. A valid inequality is deduced and improved with the setup time consideration. An relaxed IP model with valid inequality can provide a primary cable assignment scheme and lower bound to the scheduling problem. A SS algorithm is proposed to solve the problem. At last, the numerical experiments is carried out which demonstrates that the proposed solution approach is effective and efficient.
\end{abstract}

Keywords-parallel machine; scheduling; Scatter Search; integer programming

\section{INTRODUCTION}

A typical cable and cable harness manufacturer usually has two or more production lines and each of them can continually draw out cables[1]. To meet the multi-species, small quantities, and short-period development tends, each cable production line is usually designed to fit to nearly all cable products but prefers specific cable varieties to others. That is to say, the production cost and time of the same cable product can vary with different production lines. To obtain more profits, the parallel production lines need to be scheduled appropriately to deal with the accepted product orders at the cost as small as possible. In our considered scenario, the product orders can be delayed or excluded in the problem horizon at different penalty costs and some technological requirements need to be taken into accounts.

The scheduling problem can be viewed as a special parallel machine scheduling problem which has been a lot of related researches published. In 1970s, a series of researches have ever focused on problem complexity analysis [2]-[5]. And kinds of algorithms have been developed to solve the parallel machine scheduling problems of NP-hardness, including the intelligent optimization algorithm, such as Tabu Search[6], Simulated Annealing[8], Genetic Algorithm[8]\&[9], and mathematic optimization algorithms, such as Branch and Bound[10], Dynamic Programming[11], Column Generation[12].

It is worth noting that a hybrid mixed integer linear programming/constraint programming (MILP/CP) approach has been proposed to solve a class of parallel ma-chine scheduling problem[13] [14] which were similar to the scheduling problem in this paper.

\section{Problem Description AND MAthematicAL MODEL}

\section{A. The Problem Description}

In the cable production line, cables are continuously produced coil by coil[1]. A cable coil will be called "cable" and taken as a job in the scheduling problem. The cable requires different processing time and cost on different production lines because the lines are configured to different product preference. The setup time is also needed and depends on the processing sequence. The considered cables are given different tardiness penalty. The scheduling objective, to be minimized, includes two parts: the total processing costs and total tardiness penalties.

Another consideration is the time window constraint. Each cable has a release time due to the upstream process. And most cables have the contracted delivery times which arise the due dates.

\section{B. The Mixed Integer Linear Programming Model}

To simplify the problem model, we introduce a dummy production line to deal with the ignored cable (or tardy cable). On the dummy line, each cable has a zero pro-cessing time and a processing cost equal to the tardiness penalty which is typically larger than the processing costs on the real production lines.

The following notations will be referred as the problem parameter.

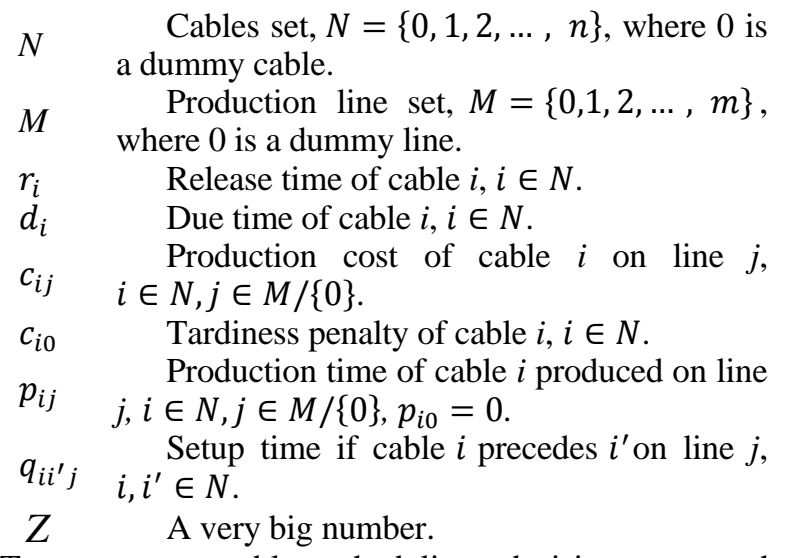

To represent cable scheduling decisions over the production lines, we introduce the following decision variables: 
MILP model, but also work for the heuristic approach. The

$y_{i j}=\left\{\begin{array}{l}0, \text { if cable } i \text { is produced on line } j ;, i \in N, j \in M . \\ 1, \text { otherwise. }\end{array}\right.$ $x_{i i^{\prime} j}$

$=\left\{\begin{array}{l}0, \text { if cable } i^{\prime} \text { is produced imediatly after } i \text { on line } j ; \\ 1, \text { otherwise. }\end{array}\right.$

$i, i^{\prime} \in N, j \in M$

$S_{i} \quad$ Start time of producing cable $i, i \in N$.

$C_{i} \quad$ Complete time of cable $i, i \in N$.

A mixed integer linear programming model can be formulated to describe the parallel cable production lines scheduling problem.

$$
\begin{aligned}
& \operatorname{Min} \sum_{i \in N \backslash\{0\}} \sum_{j \in M} c_{i j} y_{i j} \\
& \text { s.t. } \\
& \sum_{j \in M} y_{i j}=1, \quad i \in N \backslash\{0\} \\
& \sum_{i \in N, i \neq i^{\prime}} x_{i i^{\prime} j}=y_{i^{\prime} j}, \quad i^{\prime} \in N \backslash\{0\}, j \in M \\
& \sum_{i^{\prime} \in N, i^{\prime} \neq i} x_{i i^{\prime} j}=y_{i j}, \quad i \in N \backslash\{0\}, j \in M \\
& \sum_{i^{\prime} \in N \backslash\{0\}} x_{0 i^{\prime} j} \leq 1, j \in M \\
& S_{i} \geq r_{i}, i \in N \backslash\{0\} \\
& C_{i} \leq d_{i}, i \in N \backslash\{0\} \\
& S_{i^{\prime}}-\left(C_{i}+\sum_{j \in M} x_{i i^{\prime} j} q_{i i^{\prime} j}\right) \geq Z\left(\sum_{j \in M} x_{i i^{\prime} j}-1\right) \\
& i, i^{\prime} \in N \backslash\{0\} \\
& C_{i}-S_{i} \geq \sum_{j \in M} p_{i j} y_{i j}, \quad i \in N \backslash\{0\} \\
& x_{i i^{\prime}{ }^{\prime}}, y_{i j} \in\{0,1\}, \quad i, i^{\prime} \in N, j \in M \\
& S_{i}, C_{i} \geq 0, \quad i \in N
\end{aligned}
$$

The objective function (1) of the model is to minimize the total production costs and tardiness penalty costs. Constraint (2) ensures a production line assigned to each cable. Constraints (3) and (4) ensure that each cable has one preceding cable and one succeeding cable, respectively. Constraint (5) states that each line has at most one starting cable, which implies that there are at most $\mathrm{m}$ available lines. Constraints (6) - (9) are the time window constraints. Constraints (10) and (11) provide value ranges for the variables

\section{VALID INEQUALITIES AND LOWER BOUND FOR THE PROBLEM}

The valid inequality, reflecting the problem features, can not only improve the resolving efficiency of the formulated

following inequality comes from the existing research on the parallel machine scheduling ${ }^{[13][14]}$

- Production capacity inequality:

$$
\begin{gathered}
\sum_{i \in \Psi\left(r_{i^{\prime}}, d_{i^{\prime \prime}}\right)} y_{i j} p_{i j} \leq d_{i^{\prime \prime}}-r_{i^{\prime}}, i, i^{\prime} \in N \text { and } d_{i^{\prime \prime}}>r_{i^{\prime}} \\
j \in M /\{0\}
\end{gathered}
$$

where $\Psi\left(r_{i^{\prime}}, d_{i^{\prime \prime}}\right)=\left\{i \mid r_{i} \geq r_{i^{\prime}}\right.$ and $\left.d_{i} \leq d_{i^{\prime \prime}}, i \in N\right\}$ includes all cables that need to be produced in time window $\left[r_{i^{\prime}}, d_{i^{\prime \prime}}\right]$.

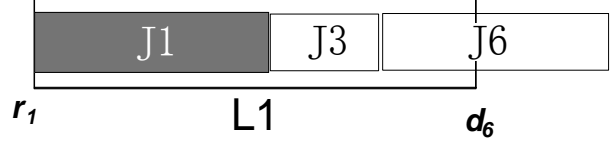

Figure 1. Cable assignments excluded by the valid inequalities.

For the case illustrated in Figure $1, \Psi\left(r_{1}, d_{6}\right)=$ $\{1,3,6\}$, that is to say that the given time windows of cable $\mathrm{J} 1, \mathrm{~J} 2$ and $\mathrm{J} 3$ are all included in time window $\left[r_{1}, d_{6}\right]$. Cable combination $J 1, J 3, J 6$ can be excluded from L1 through inequality (12), because their total processing time is larger than $d_{6}-r_{1}$.

Since the setup time needs to be considered in our problem, let $P_{i j}=p_{i j}+\operatorname{Min}\left\{q_{i^{\prime} i j} \mid i^{\prime} \in N\right\}$ replace $p_{i j}$ in formula (12). That is

$$
\begin{gathered}
\sum_{i \in \Psi\left(r_{i^{\prime}}, d_{i^{\prime \prime}}\right)} y_{i j} P_{i j} \leq d_{i^{\prime \prime}}-r_{i^{\prime}}, \\
\forall i, i^{\prime} \in N \text { and } d_{i^{\prime \prime}}>r_{i^{\prime}}, j \in M \backslash\{0\}
\end{gathered}
$$

Inequality (13) is obviously tighter than inequality (12).

An existing MILP/CP approach for the classic parallel machines scheduling problem can also be applied to this scheduling problem of the small-scale (see literature [13]\&[14] for more details), through taking model (1), (2) \& (13) as the master problem. For the larger problem instances, integer programming (IP) model (1), (2) \& (13) can provide a lower bound.

\section{THE SCATTER SEARCH FOR THE PROBLEM}

Scatter search (SS) is a metaheuristics based on evolution population. Its search strategy considers both the intensification and diversification of solution pool (population) and thus performs well at global search[15] [16]. Generally, a desirable SS can converge quickly at the same time avoiding premature convergence to low-quality solutions. In practice, it needs a good balance between computational time and solution quality.

The algorithm framework of the SS is shown in Figure 1 and its steps are listed as following:

- Initialize the solution pool $\mathrm{P}$ - Generate $|P|$ different solutions by repeatedly using the randomized heuristic method. Populate $P$ with those generated 
solutions. Sort the solutions in $P$ by their objective value in ascending order.

- $\quad$ Build RefSet - Build RefSet $=\left\{x^{1}, \ldots, x^{b 1}\right\}$ with the first $b_{1}$ solutions in $P$ and remove them from $P$ except the best one. For each solution in $P$, compute the minimum of distances to the solutions in RefSet through the distance measurement method. Add the solution with maximum of the minimum distances to RefSet and remove it from $P$. Repeat this process for $b_{2}$ times and then RefSet $=\left\{x^{1}, \ldots, x^{b}\right\}$ where $b=b_{1}+$ $b_{2}$.

- Combine solutions based on RefSet - Generate $b(b-1)$ solutions by applying the solution combination method to each 2-solution array in RefSet, because array $\left\langle x^{1}, x^{2}\right\rangle$ and $\left\langle x^{2}, x^{1}\right\rangle$ can create two different solutions. Improve those solutions by the improvement method.

- Update $P$ - Select some good solutions from those new solutions to populate $P$ or replace some worse solutions in $P$. Sort the solutions in $P$ by the objective value. If most solutions in $P$ are same as those in the last iteration, $P$ would be assumed to converge.

- $\quad$ Reinitialize $P$ - Just like the above initialization step except that include the best solution as far, the first one in $P$.

- Termination - The approach terminates if the best solution has retained without being updated for a number of iterations.

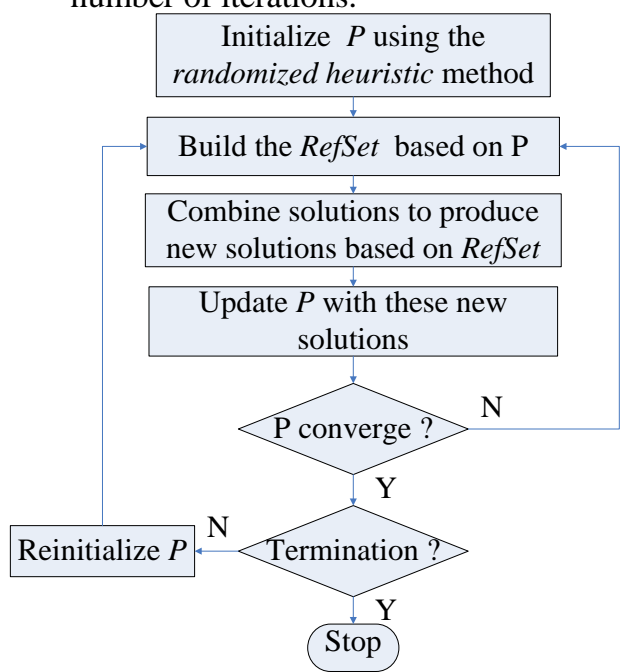

Figure 2. The algorithm framework of Scatter Search for the problem.

\section{A. The Randomized Solution Generator}

A randomized solution generator, same as that was proposed in Tang, Ren \& Yang[17], is implemented to generate diverse trial solutions. In addition, it can provide a preliminary cable assignment scheme to solve IP model (1), (2) and (13). The ILOG Cplex, an optimization software for LP and MIIP model, is employed to solve the model. The cable assignment scheme can be extended to a solution through applying the earliest due date first (EDD) rule to each production line.

\section{B. Distance Measurement Method Between Solutions}

An accurate inter-solution distance (or difference) measurement is important to control the diversity of the reference set (RefSet). Due to the cable assignment over the production lines is the main factor to affect the objective function, the ratio of number of same cable assignments to total number of cables is taken as the distance measurement.

\section{The Improvement Method}

A simple local search, based on the swap and insert neighborhoods, is applied to all solutions in all generations while a local search, based on a variable-depth cycle exchange neighborhood (see Luo and Tang[18] for more details), is applied to solutions when the best solution found so far has not changed for 10 generations.

The swap neighborhood is obtained through exchanging the assigned production lines of each pair of genes in the given chromosome. The production orders should be exchanged if the pair of genes is corresponding to the same line. Similar to the swap neighborhood, the insert neighborhood involves with inserting a cable to another line or a different position in the same line. The technical constraint has a higher priority than the EDD ruler when they conflict.

\section{The Solution Coding and Combination Method}

The vector-group coding method [19] is adopted to facilitate the combination operation. In the coding method, a solution is constructed using a sequence of two-dimension vectors as follows:

$$
\left[\begin{array}{llll}
g_{1} & g_{2} & \ldots & g_{n}
\end{array}\right]=\left[\begin{array}{cccc}
h_{1} & h_{2} & \ldots & h_{n} \\
k_{1} & k_{2} & \ldots & k_{n}
\end{array}\right]
$$

where $\left[\begin{array}{l}h_{i} \\ k_{i}\end{array}\right]$ represents the $i$ th cable assignment in the solution, $h_{i} \in N$ is a cable, $k_{i} \in M$ is the production line to process cable $h_{i}$ and $\left\{h_{1}, h_{2}, \ldots, h_{k}\right\}=N \backslash\{0\}$. The cables assigned to the same line will be processed in order of the vector-group. The dummy production line is considered in the coding scheme to deal with the tardy jobs (cables).

Based on the vector-group coding scheme, we adopt one-point extended order to combine a new solution. Let solutions Sol1 $=\left[\begin{array}{llll}g_{1} & g_{2} & \ldots & g_{n}\end{array}\right]$ and Sol2 $=\left[\begin{array}{llll}g_{1}^{\prime} & g_{2}^{\prime} & \ldots & g_{n}^{\prime}\end{array}\right]$ serve as the source solutions. A random integer $\lambda$ with $1 \leq \lambda<m$ is first generated to decide the boundary point. The new combination solution is $\left[g_{1} g_{2} \ldots g_{\lambda} g_{\lambda+1}^{\prime} \ldots g_{n}^{\prime}\right]$ if $\left\{g_{1}, g_{2}, \ldots, g_{\lambda}\right\}$ and $\left\{g_{\lambda+1}^{\prime}, \ldots, g_{n}^{\prime}\right\}$ do not includes a common cable; otherwise, discard the elements with common cable in Sol2 and insert the elements corresponding to the lost cables in the in Sol2, since the number of common cables must be equal to the number of lost ones.

The combination method can generate different chromosome to reverse the Sol1 and Sol2.

\section{COMPUTATIONAL EXPERIMENTS}

Base on the above model and algorithm, we developed the involved program and tested it under VC++ 2010 
environment. The lower bound was obtained through solving the IP model with ILOG Cplex. The experiments were all performed on a computer with Win 7 operation system and $2.8 \mathrm{GHz}$ Intel 2 Core CPU and 4 GB RAM.

The SS parameters for all problems were set to be the same: referred solution set size $b=b_{1}+b_{2}=12+8.12$ problem instances came from a practical cable production enterprise with four available parallel production lines and one reserve line. The production cost and times and setup times were all empirical data. To test the SS approach thoroughly, a series of problem instances of 6 different sizes were generated through imitating the practical data.

The computing result for the 12 practical problem instances was list in Table I. Each problem had 4 production lines and the problem sizes were the considered number of cables. In Table I, column $L B$ showed the lower bound of the problem instance. The lower bound was obtained through solving the integer programming (IP) model (1), (2) \& (13) with ILOG CPLEX (a commercial optimization software). The average relative gap is $3.75 \%$ and the average CPU-s is 75 .

TABLE I. EXPERIMENT RESULT FOR THE PRACTICAL

\begin{tabular}{|c|c|c|c|c|c|}
\hline \multirow{2}{*}{ Problem } & \multirow{2}{*}{ Size } & \multicolumn{2}{|c|}{ Objective value } & \multirow[b]{2}{*}{ Gap (\%) } & \multirow[b]{2}{*}{$\begin{array}{c}\text { CPU } \\
\text { (Seconds) }\end{array}$} \\
\hline & & SS & $L B$ & & \\
\hline 1 & 43 & 297 & 293 & 2.41 & 33 \\
\hline 2 & 44 & 353 & 349 & 2.28 & 57 \\
\hline 3 & 53 & 364 & 350 & 4.96 & 116 \\
\hline 4 & 53 & 376 & 371 & 2.43 & 12 \\
\hline 5 & 57 & 414 & 402 & 4.04 & 47 \\
\hline 6 & 61 & 515 & 506 & 2.84 & 44 \\
\hline 7 & 64 & 555 & 544 & 3.07 & 14 \\
\hline 8 & 67 & 581 & 561 & 4.65 & 17 \\
\hline 9 & 73 & 612 & 579 & 6.66 & 232 \\
\hline 10 & 79 & 647 & 632 & 3.38 & 40 \\
\hline 11 & 87 & 594 & 585 & 2.52 & 221 \\
\hline 12 & 89 & 693 & 661 & 5.80 & 68 \\
\hline Average & -- & -- & -- & 3.75 & 75 \\
\hline
\end{tabular}

Table II listed the computational results for the generated 6 problem sizes. It was observed that the computation times (CPU time) increased with the number of cables but the average solution Gap was relatively stable.
TABLE II. THE AVERAGE COMPUTING RESULT FOR THE GENERATED 6 GROUP OF PROBLEM INSTANCES.

\begin{tabular}{|l|c|c|}
\hline Size & Gap & CPU time (Seconds) \\
\hline $40 \times 3$ & 2.43 & 45 \\
\hline $60 \times 3$ & 2.26 & 47 \\
\hline $80 \times 3$ & 4.98 & 128 \\
\hline $40 \times 4$ & 2.41 & 16 \\
\hline $60 \times 4$ & 3.93 & 44 \\
\hline $80 \times 4$ & 2.95 & 146 \\
\hline Average & 3.16 & 70 \\
\hline
\end{tabular}

In those experiments, the IP model can provide the primary cable assignment scheme to the SS as well as the lower bound to evaluate the solution quality. From the computation results, we have the following observations.

- All the problem instances could be resolved in acceptable computing times (no more than $5 \mathrm{~min}$ ), as demonstrated in Table I\&II. The proposed SS approach performs efficient.

- - As shown in Table I\&II, the computation times increases with the problem size but the solution quality is relatively stable. It implies that the solution quality is seldom affected by the problem size and the computing time depends on the problem size.

- - The IP model with the validate inequality can provide the lower bound to evaluate the solution quality. The relative small gap implies the effectiveness of the lower bound as well as the solution approach.

\section{CONCLUSION}

In this paper, a cable parallel production lines scheduling problem was first formulated into a mixed integer programming model, considering setup time and time window. A valid inequality was deduced and improved with the setup time consideration which provided a primary cable assignment scheme and lower bound for the scheduling problem. An SS algorithm was proposed to solve the problem. At last, the numerical experiments were carried out which demonstrated that the proposed solution approach was effective and efficient.

\section{ACKNOWLEDGMENT}

This research was supported by National Natural Science Foundation of China (71201105) and China Postdoctoral Science Foundation (2013M530947). 


\section{REFERENCES}

[1] Niroomand S., Vizvari B.: Exact Mathematical Formulations and Metaheuristic Algorithms for Production Cost Minimization: A Case Study Of The Cable Industry. International Transactions in Operational Research. 22(3), 519-544 (2015)

[2] Graham R.L., Lawler E.L., Lenstra J.K., Rinnooy Kan A.H.G.: Optimisation and Approximation in Deterministic Sequencing and Scheduling: A Survey. Annals of Discrete Mathematics. 5, 287-326 (1979).

[3] Karp R.M.: Reducibility Among Conbinatorial Problems. In: Proceedings of Complexity of Computer Computations, New York, US, (1972).

[4] Bruno J.L., Coffman E.G. Sethi J.R.: Scheduling Independent Tasks To Reduce Mean Finishing Time. ACM Communications. 17, 382387 (1974).

[5] Horowitz E., Sahni S.: Exact and Approximate Algorithms for Scheduling Non-Identical Processors. Journal of the Association for Computing Machinery. 2(23), 317-327 (1976).

[6] Bozorgirad M.A., Logendran R.: A comparison of local search algorithms with population-based algorithms in hybrid flow shop scheduling problems with realistic characteristics. International Journal of Advanced Manufacturing Technology. DOI 10.1007/s00170-015-7650-9, (2015).

[7] Xiao J., Yang H., Zhang C., Zheng L.: A hybrid Lagrangiansimulated annealing-based heuristic for the parallel-machine capacitated lot-sizing and scheduling problem with sequencedependent setup times. Computers \& Operations Research. 63(2):7282, (2015).

[8] Bao J., Wang Q., Xu A.: A Steerable GA Method for Block Erection of Shipbuilding in Virtual Environment. Lecture Notes in Computer Science. 8683:286-293 (2014).
[9] Zhao C., Tang H.: Parallel machines scheduling with deteriorating jobs and availability constraints. Japan Journal of Industrial \& Applied Mathematics. 31(3): 501-512, (2014).

[10] Liaw C.: A branch-and-bound algorithm for identical parallel machine total tardiness scheduling problem with preemption. Journal of Industrialand Production Engineering. DOI:10.1080/21681015.2016.1147088,(2015).

[11] Sahni S.: Alogirthms for Scheduling Independent Tasks. Journal of the Association for Computing Machinery, 23: 116-127 (1976).

[12] Dong M.: Parallel machine scheduling with limited controllable machine availability. International Journal of Production Research, 51(8):2240-2252, (2013).

[13] Maravelias C.T., Grossmann I.E.: A Hybrid MILP/CP Decomposition Approach For The Continuous Time Scheduling Of Multipurpose Batch Plants. Computers and Chemical Engineering. 28: 1921-1949 (2004).

[14] Ren H., Liu S.: Study on the Hybrid IP/CP Approach for a MultiMachine Assignment Scheduling Problem. In: Proceedings of the International Conference on Management and Engineering, Shanghai, China (2014).

[15] Glover F.: A Template For Scatter Search And Path Relinking. Lecture Notes in Computer Science, 1363:1-51, (1998).

[16] Guo Q., Tang L., Glover F.: A scatter search based heuristic for the balancing of parallel assembly lines. Proceedings of the 48th IEEE Conference on Decision and Control. 6256 - 6261, (2009).

[17] Tang L., Ren H., Yang Y.: Reheat Furnace Scheduling With Energy Consideration. International Journal of Production Research. 63(6), 1642-1660 (2015).

[18] Luo J., Tang L.: A New ILS \& SS Algorithm for Parallel-machine Scheduling Problem [J], ACTA ELECTRONICA SINICA. 31(6), 917-924 (2005).

[19] Yin W., Liu M., Wu C.: A New Genetic Algorithm for Parallel Machine Scheduling with Process Constraint. ACTA ELECTRON ICA SINICA. 29(11),1482-1485 (2001). 